\title{
Multi-scale magnetic field intermittence in the plasma sheet
}

\author{
Z. Vörös ${ }^{1}$, W. Baumjohann ${ }^{1}$, R. Nakamura ${ }^{1}$, A. Runov ${ }^{1}$, T. L. Zhang ${ }^{1}$, M. Volwerk ${ }^{1}$, H. U. Eichelberger ${ }^{1}$, A. Balogh ${ }^{2}$, \\ T. S. Horbury ${ }^{2}$, K.-H. Glaßmeier ${ }^{3}$, B. Klecker ${ }^{4}$, and H. Rème ${ }^{5}$ \\ ${ }^{1}$ Institut für Weltraumforschung der ÖAW, Graz, Austria \\ ${ }^{2}$ Imperial College, London, UK \\ ${ }^{3} \mathrm{TU}$ Braunschweig, Germany \\ ${ }^{4}$ Max-Planck-Institut für extraterrestrische Physik, Garching, Germany \\ ${ }^{5}$ CESR/CNRS, Toulouse, France
}

Received: 27 September 2002 - Revised: 14 December 2002 - Accepted: 29 January 2003

\begin{abstract}
This paper demonstrates that intermittent magnetic field fluctuations in the plasma sheet exhibit transitory, localized, and multi-scale features. We propose a multifractal-based algorithm, which quantifies intermittence on the basis of the statistical distribution of the "strength of burstiness", estimated within a sliding window. Interesting multi-scale phenomena observed by the Cluster spacecraft include large-scale motion of the current sheet and bursty bulk flow associated turbulence, interpreted as a cross-scale coupling (CSC) process.
\end{abstract}

Key words. Magnetospheric physics (magnetotail; plasma sheet) - Space plasma physics (turbulence)

\section{Introduction}

The study of turbulence in near-Earth cosmic plasma is important in many respects. Turbulence, being in its nature a multi-scale phenomenon, may influence the transfer processes of energy, mass and momentum on both MHD and kinetic scales. Vice versa, turbulence can be driven by instabilities, such as magnetic reconnection or current disruption (Tetreault, 1992; Angelopoulos et al., 1999a; Klimas et al., 2000; Chang et al., 2002; Lui, 2002).

The understanding of intermittence features of fluctuations is fundamental to turbulence. Intermittence simply refers to processes which display "sporadic activity" during only a small fraction of the considered time or space. This is also the case in non-homogeneous turbulence, where the distribution of energy dissipation regions is sporadic and probability distributions of measurable quantities are long-tailed with significant departures from Gaussianity. Rare events forming the tails of probability distribution functions, however, carry a decisive amount of energy present in a process (Frisch, 1995).

Substantial experimental evidence exists for the occurence of intermittent processes within the plasma sheet. Baumjo-

Correspondence to: Z. Vörös (Zoltan.Voeroes@oeaw.ac.at) hann et al. (1990) showed that within the inner plasma sheet inside of $20 R_{E}$, high-speed short-lived ( $\left.\sim 10 \mathrm{~s}\right)$ plasma flows are rather bursty. Angelopoulos et al. (1992) noted that those flows organize themselves into $\sim 10 \mathrm{~min}$ time scale groups called bursty bulk flows (BBF). Despite the fact that BBFs represent relatively rare events (10-20\% of all measurements), they are the carriers of the decisive amount of mass, momentum and magnetic flux (Angelopoulos et al., 1999b; Schödel et al., 2001) and can, therefore, energetically influence the near-Earth auroral regions (Nakamura et al., 2001).

So far experimental evidence for real plasma sheet turbulence is not unambiguous; however, its existence is supported by the occurrence of plasma fluctuations in bulk flow velocity and magnetic field which are comparable or even larger than the corresponding mean values (Borovsky et al., 1997). Other characteristics of plasma sheet turbulence, such as probability distributions, mixing length, eddy viscosity, power spectra, magnetic Reynolds number, etc., were found to exhibit the expected features or to be in expected ranges predicted by turbulence theories (Borovsky et al., 1997). Though the amplitude of the velocity and magnetic field fluctuations increases with geomagnetic activity (Neagu et al., 2002), intense fluctuations are present independently from the level of geomagnetic activity (Borovsky et al., 1997), indicating that different sources or driving mechanisms might be involved in their generation. In fact, according to observations by Angelopoulos et al. (1999a), at least a bi-modal state of the inner plasma sheet convection is recognizable from plasma flow magnitude probability density functions: BBF-associated intermittent jet turbulence and intermittent turbulence which occurs during non-BBF (quiet background) flows. Angelopoulos et al. (1999a) have also proposed that BBF-generated intermittent turbulence can alter transport processes in the plasma sheet and may represent a way for cross-scale coupling (CSC) to take place. 

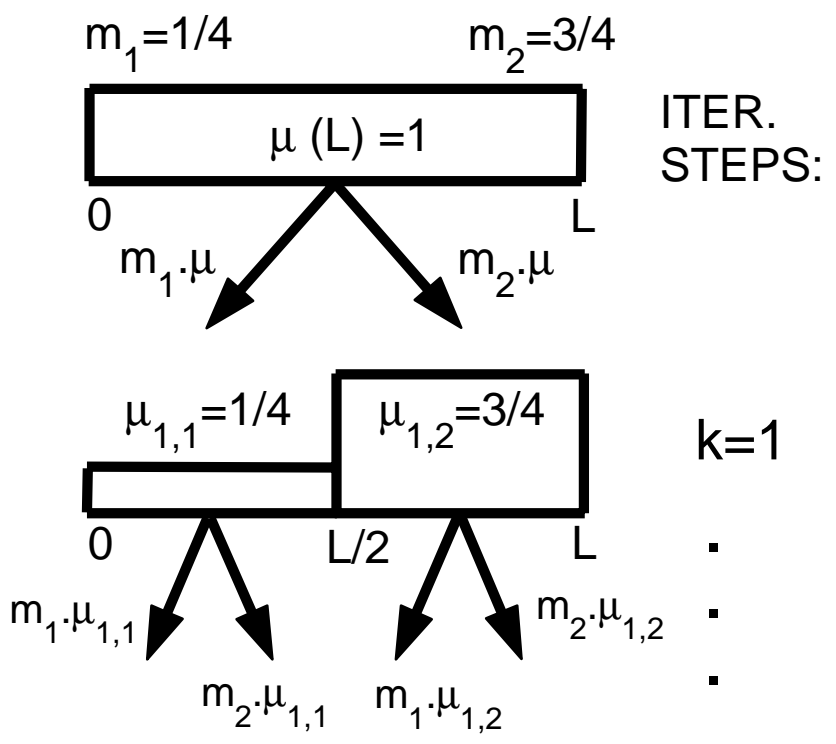

Fig. 1. Recursive construction rule for binomial distribution.

These facts call for a method which allows for analysis of both intermittence and multi-scale properties of fluctuations. In this paper we propose a multifractal technique for this purpose. Using both magnetic field and ion velocity data from Cluster, we will show that BBF-associated "magnetic turbulence" exhibits clear signatures of cross-scale energisation.

\section{Multifractal approach to turbulence}

In order to elucidate the basic assumptions of our approach we use a multinomial distribution model first and introduce a local parameter for quantification of the intermittence level on a given scale. Then, we discuss the range of potential scales over which the presence of cross-scale energisation might be experimentally demonstrable and mention some limitations regarding the availability of multipoint observations.

\subsection{Local intermittence measure $(L I M)$}

The large-scale representation of magnetotail processes by mean values of measurable quantities is useful, but can also be misleading in characterising multi-scale phenomena when quantities observed on different scales carry physically important information.

Multifractals are well suited for describing local scaling properties of dissipation fields in non-homogeneous turbulence (Frisch, 1995). Therefore, they are most suitable for a description of plasma sheet fluctuations. In nonhomogeneous turbulence, the transfer of energy from large scales to smaller scales can be conveniently modeled by a multiplicative cascade process. The distribution of energy dissipation fields on small scales exhibits burstiness and intermittence.
Let us consider a simple model example. Multinomial deterministic measures are examples of multifractals (Riedi, 1999). These consist of a simple recursive construction rule: a uniform measure $\mu(L)$ is chosen on an interval $I:[0, L]$ and is then unevenly distributed over $n>1(n-$ integer $)$ equal subintervals of $I$ using weights $m_{i} ; i=1, \ldots, n$ and $\sum_{i} m_{i}=1$. Usually $L$ is chosen to be 1 . After the first iteration we have $n$ equal subintervals, and subinterval $i$ contains a fraction $\mu(L) m_{i}$ of $\mu(L)$. Next, every subinterval and the measure on it are split in the same way recursively, having $i=1, \ldots, n^{k}$ subintervals or boxes after $k$ iteration steps and $\mu_{k, i}$ in the box $I_{k, i}$. Figure 1 shows the simplest example of a binomial distribution $(n=2)$. We note that the measure $\mu$ can be any positive and additive quantity, such as energy, mass, etc.

Figure 2a presents two distributions, $A$ and $B$, separated by a dashed vertical line in the middle. Both mimic typical bursty "time series" like a physical variable from a turbulent system, however, by construction distribution $A$ is less intermittent than distribution $B$. In both cases the same initial mass $(\mu)$ is distributed over interval $L, n=8 ; k=5$ is chosen (that is $n^{k}=32768$ boxes), but the weights $m_{i}(A)=(0.125,0.08,0.09,0.16,0.05,0.25,0.12,0.125)$ and $m_{i}(B)=(0.1,0.3,0.05,0.002,0.04,0.218,0.09,0.2)$ are different. Intermittence is larger in case $B$ (Fig. 2a) because of the larger differences between weights (if all weights were equal, the resulting distribution would become homogeneous). Our goal is to quantify this level of intermittence by multifractals. The definition of multifractality in terms of the large deviation principle simply states that a dissipation field, characterized locally by a given "strength of burstiness" $\alpha$, has a distribution $f(\alpha)$ over the considered field. It measures a deviaton of the observed $\alpha$ from the expected value $\bar{\alpha}$. The corresponding $(\alpha, f(\alpha))$ large deviation spectrum is of concave shape (Riedi, 1999).

The strength of local burstiness, the so-called coarse-grain Hölder exponent $\alpha$, is computed as

$\alpha_{i} \sim \frac{\log \mu_{k, i}}{\log [I]_{k, i}}$,

where $[I]_{k, i}$ is the size of the $k, i$-th box and equality holds asymptotically.

It is expected that, due to its multiplicative construction rule $\mu_{k, i}$ will decay fast as $[I]_{k, i} \rightarrow 0$ and $k \rightarrow \infty$. We add that $\alpha_{i}<1$ indicates bursts on all scales, while $\alpha_{i}>1$ characterizes regions where events occur sparsely (Riedi, 1999). Equation (1) then expresses the power-law dependence of the measure on resolution. Usually "histogram methods" are used for the estimation of the $f(\alpha)$ spectrum (also called rate function), so that the number of intervals $I_{k, i}$ for which $\alpha_{k, i}$ falls in a box between $\alpha_{\min }$ and $\alpha_{\max }$ (the estimated minimum and maximum values of $\alpha$ ) is computed and $f(\alpha)$ is found by regression. In this paper, however, $f(\alpha)$ spectra are estimated using the FRACLAB package which was developed at the Institute National de Recherche en Informatique, Le Chesnay, France. Here, the well-known statistical kernel 


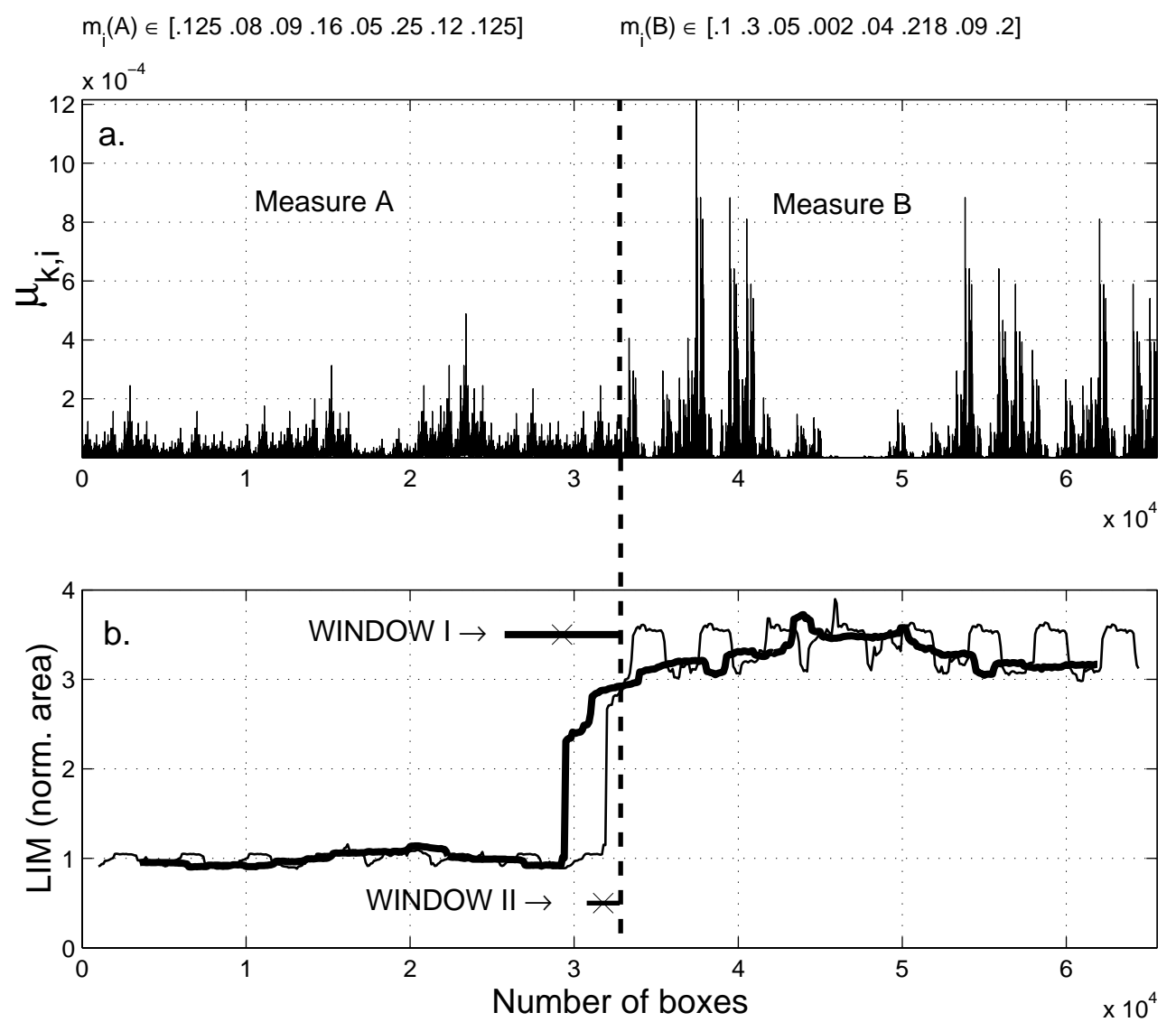

Fig. 2. (a) Two multinomial distributions: measure A is less intermittent than measure B; dashed line in the middle separates the two measures. (b) LI M estimation for two different windows I and II.

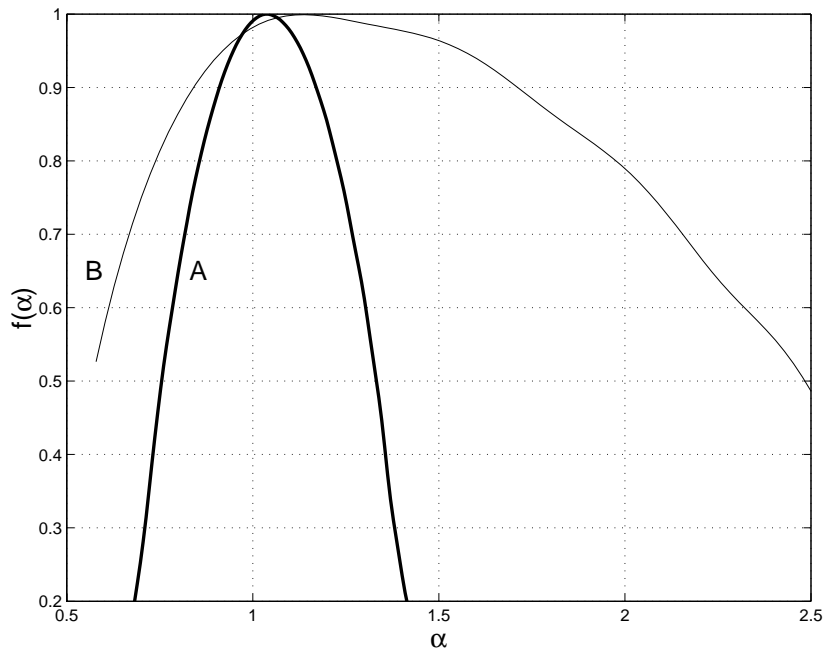

Fig. 3. Multifractal distributions for measures A and B shown in Fig. 2a.

method for density estimations is used which also yields satisfactory estimations for processes different from purely multiplicative ones (Véhel and Vojak, 1998; Canus et al., 1998).
A comparison of Figs. 2a and 3 indicates that the wider the $f(\alpha)$ spectrum is, the more intermittent the measure is. This feature was also proposed for the study of the possible role of turbulence in solar wind - magnetosphere coupling processes (Vörös et al., 2002), and this feature will be used to describe magnetic field intermittence in the plasma sheet.

In order to gain appropriate information about the time evolution of intermittence from real data, we estimate $f(\alpha)$ within sliding overlapping windows $W$ with a shift $S \ll W$. In our model case the time axis is represented by increasing the number of subintervals $I_{k, i} . L I M$ is introduced as the total area under each $f(\alpha)$ curve within a window $W$, divided by the mean area obtained from the measurements along the reference measure $A$. Actually, $L I M(A)$ fluctuates around 1 , due to errors introduced by finite window length. For measures exhibiting a higher level of intermittence than the reference measure $A, L I M>1$. Figure $2 \mathrm{~b}$ shows that for measures $A$ and $B$, the different levels of intermittence are properly recognized by $L I M$. Estimations based on a larger window (Window I: $W=7000$ boxes, $S=100$ boxes) are more robust, but a smaller window (Window II: $W=2000$ boxes, $S=100$ boxes) allows for a better localization of the transition point between measures $A$ and $B$ (thick line in the middle of Fig. 2a). 


\subsection{Multi-scale LIM}

Deterministic multinomial measures are self-similar in the sense that the construction rule is the same at every scale. Real data are more complex. Physical processes may have characteristic scales or may distribute energy differently over some ranges of scales. In order to study BBF-associated magnetic turbulence on both large and small scales, we introduce a "time scale" $\tau$ through differentiation

$\delta B_{x}(t, \tau)=B_{x}(t+\tau)-B_{x}(t)$.

Throughout the paper the GSM coordinate system is used in which the $\mathrm{x}$ axis is defined along the line connecting the center of the Sun to the center of the Earth. The origin is defined at the center of the Earth and is positive towards the Sun. Then, a normalized measure at a time $t_{i}$ is given by

$\mu_{B_{x}}\left(t_{i}, \tau\right)=\frac{\delta B_{x}^{2}\left(t_{i}, \tau\right)}{\sum_{i} \delta B_{x}^{2}\left(t_{i}, \tau\right)}$.

We have to mention, however, some essential limitations of this approach when a separation of spatial and temporal variations is eventually addressed. A time series obtained from a single spacecraft can be used for mapping the spatial structure of turbulence, using the so-called Taylor's hypothesis, if the spatial fluctuations on a scale $l$ pass over the spacecraft faster than they typically fluctuate in time. In the plasma sheet this is probably the case during fast BBFs (Horbury, 2000). Otherwise, Taylor's hypothesis may not be completely valid. Instead of Eq. (2) a real two-point expression, $\delta B_{x+l}(t)=B_{x+l}(t)-B_{x}(t)$ could be used, where $l$ is a distance between Cluster spacecraft. The corresponding $L I M$, however, strongly fluctuates in a variety of cases (not shown), presumably due to mapping of physically different and structured regions by individual Cluster satellites. We postpone this kind of multi-point observations to future work.

Nevertheless, Angelopoulos et al. (1999a) noticed that some characteristics of turbulence estimated from single point measurements are equivalent to ones from two-point measurements, for distances at or beyond the upper limit of the inertial range in which case Eq. (2) can be used efficiently. Borovsky et al. (1997) estimated the lower limit of the inertial range to be about ion gyroperiod time scales ( $\sim 10 \mathrm{~s}$ in plasma sheet), over which a strong dissipation of MHD structures is expected. The upper limit of inertial range (largest scale) was identified by a plasma sheet convection time scale or by inter-substorm time scale, both of the order of $5 \mathrm{~h}$. As known, inertial range refers to a range of wave numbers (or corresponding scales) over which turbulence dynamics is characterized by zero forcing and dissipation (Frisch, 1995). Recent theoretical and experimental work shows, however, that inertial range cascades might be exceptional. In a large variety of turbulent flows, rather bidirectional direct coupling (or cross scale coupling - CSC), due to nonlinearity and nonlocality between large and small scales, exists (Tsinober, 2001). While the large scales are determined by velocity fluctuations, the small scales are represented by the field of velocity derivatives (vorticity, strain).

\section{Data analysis}

\subsection{General considerations}

In this paper we analyse intermittence properties of $22 \mathrm{~Hz}$ resolution magnetic field data from the Cluster (CL) fluxgate magnetometer (FGM) (Balogh et al., 2001) and compare those characteristics with the spin-resolution $(\sim 4 \mathrm{~s})$ velocity data from the Cluster ion spectrometry (CIS/CODIF) experiment (Rème et al., 2001).

Compared with the previous model example, the estimation of the $L I M$ for the $B_{X}$ component of the magnetic data was somewhat different. First of all, we calculated $\operatorname{LIM}(t, \tau)$ for different time scales $\tau$. In the optimal case energization through a cascading process should appear on different scales that are time shifted, that is the large scales should become energized first and the small scales later. We found, however, that on various scales LIM fluctuates strongly (not shown) and using this approach it would be hard to identify an energy cascading process within an inertial range of scales. This was not unexpected, because cascade models are treated in Fourier space (wave vector space), whereas our approach represents a pure time-domain analysis method (though the magnetic field data itself already contain some spatial information), so the individual scales have rather different meanings. Also, nonlinear and nonlocal direct interactions between scales may prevent experimental recognition of cascades.

Therefore, we decided to estimate $L I M$ on several scales around $40 \mathrm{~s}$, which is considered to be a typical large scale of BBF velocity fluctuations, and compute the average $L I M_{L}$ (subscript $L$ reads as large scale) from the corresponding $f(\alpha)$ spectra. BBF events usually last several minutes (Angelopoulos et al., 1992), however, if $\tau$ is chosen to be several minutes long, the corresponding window length $\mathrm{W}$ should be even several times longer, which would make measurements of the non-stationary features of intermittence almost impossible.

A typical small scale was chosen experimentally. We looked for a $\tau$ (Eq. 2) which reflects the small-scale changes of the intermittence level properly. We found that fluctuations on time scales larger than a few seconds already exhibit similar intermittence properties as on scales around $40 \mathrm{~s}$. In fact, the majority of bursty flows may remain uninteruptedly at high speed levels for a few seconds (Baumjohann et al., 1990). Therefore, we considered time scales around $0.4 \mathrm{~s}$ as small ones (two orders less than the chosen large scale) and the corresponding intermittence measure reads as $L I M_{S}$. This time scale may already comprise some kinetic effects. The use of $22-\mathrm{Hz}$ resolution magnetic data from the FGM experiment on such small time scales implies the problem of different transfer functions for high and low frequencies. Corrections introduced by appropriate filtering had no effect on the $L I M$ estimations. 

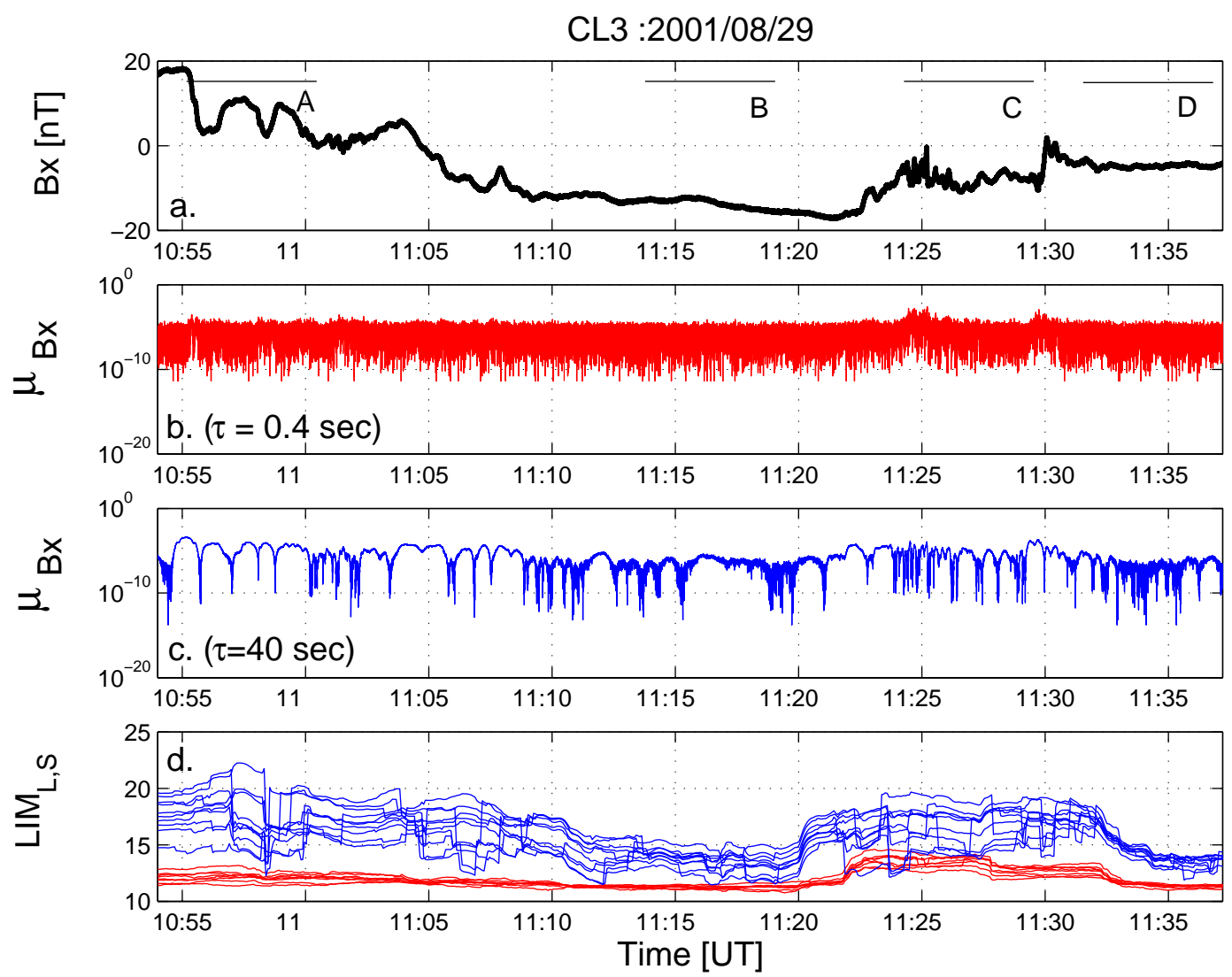

Fig. 4. (a) Magnetic field $B_{X}$ component measured by Cluster 3; (b) the associated measure computed by using Eqs. (2) and (3) on small scales (red colour); (c) the same on large scales (blue colour); (d) small and large scale $L I M_{L, S}$.

\subsection{Event overview and $L I M$ analysis}

The events we are interested in occured between 10:55 and 11:35 UT on 29 August 2001 (Fig. 4a), when CL was located at a radial distance of about $19.2 R_{E}$, near midnight. In the following the relatively "quiet" time period from $11: 15$ to 11:20 UT will be used as a reference level for both $L I M_{L}$ and $L I M_{S}$ estimations. It means that during this time period the $L I M_{L, S}$ mean values equal 1 .

The current sheet structure and movement during 10:5511:07 UT has been studied by Runov et al. (2002). Only the $B_{X}$ component from CL 3 will be evaluated. During the chosen interval, CL 3 was located approximately $1500 \mathrm{~km}$ south of the other three spacecraft. CL traversed the neutral sheet from the Northern $\left(B_{X} \sim 20 \mathrm{nT}\right)$ to the Southern Hemisphere $\left(B_{X} \sim-15 \mathrm{nT}\right)$, then $B_{X}$ approached $B_{X} \sim 0$ again (Fig. 4a). The correspondingly normalized small-scale ( $\tau=0.4 \mathrm{~s})$ and large-scale $(\tau=40 \mathrm{~s})$ measures (Eqs. 2 and 3 ) are depicted by red and blue curves in Fig. 4 b and c, respectively. In fact, Eq. (2) represents a high-pass or low-pass filter for properly chosen time shifts $\tau$. Therefore, Fig. 4b (4c) shows an enhanced level of small-scale (large-scale) fluctuations when high-frequency (low-frequency) fluctuations are present in Fig. 4a. $L I M_{L, S}$ were computed as a changing area under $f(\alpha)$ multifractal distribution curves over the interval $\alpha \in\left(1, \alpha_{\max }\right)$ and within the sliding window $W=318 \mathrm{~s}$. The time shift is $S=4.5 \mathrm{~s}$. These parameters were chosen such that the opposing requirements for the stability of LIM estimations (wide window needed) and for time-localization of non-stationary events (narrow window needed) were matched. Considering the whole area under the $f(\alpha)$ curves, i.e. estimating $L I M$ over $\alpha \in\left(\alpha_{\min }, \alpha_{\max }\right)$ as in the previous section (model case), would be also possible. This gives, however, the same qualitative results. During intervals of changing intermittence levels, mainly the right wing of $f(\alpha)$ changes. Therefore, we estimated LIM over the interval $\alpha \in\left(1, \alpha_{\max }\right)$. Figure $4 \mathrm{~d}$ shows 10 red curves of $L I M_{S}(t, \tau)$ computed for $\tau \in(0.3,0.5) \mathrm{s}$, and 10 blue curves for $\tau \in(30,50) \mathrm{s}$. Obviously, $L I M_{L}$ and $L I M_{S}$ exhibit quite different courses, and we will analyse the differences in more detail.

First, we examine the $f(\alpha)$ multifractal spectra. Windows A, B, C and D in Fig. 4a indicate periods during which distinct physical phenomena occured. The differences are evident from the magnetic field $B_{X}$, measures $\mu_{B_{x}}$ and $L I M_{L, S}$ evolution over time (Figs. $4 \mathrm{a}-\mathrm{d}$ ). We focus mainly on an interval between 11:23 UT and 11:33 UT, in which both $L I M_{L}$ and $L I M_{S}$ have increased values. Period $\mathrm{C}$ is during this interval. We contrast this interval with 10:55 to 11:10 UT, at the beginning of which a wavy flapping motion or an expansion- 

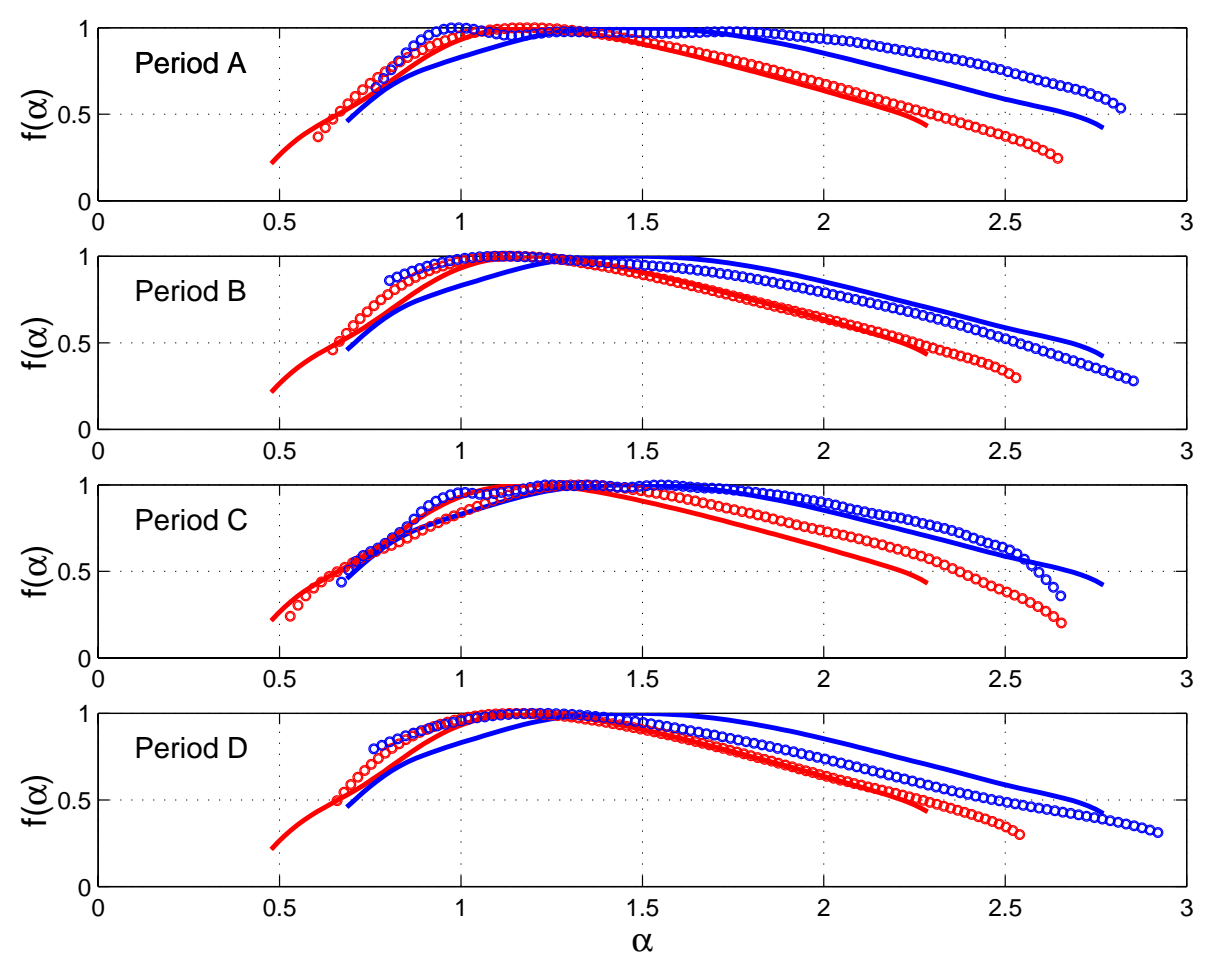

Fig. 5. Multifractal spectra for periods A-D shown in Fig. 4a (red circles: small scales, blue circles: large scales); continuous curves with the same colour code correspond to average multifractal spectra estimated for the whole interval from 10:55 to 11:35 UT.

contraction of the current sheet is observed (Period A) with a characteristic time scale of 70-90 s (Runov et al., 2002). Periods $\mathrm{B}$ and $\mathrm{C}$ represent quiet intervals with different $B_{X}$ values. The corresponding $f(\alpha)$ spectra are depicted by red and blue circles in Fig. 5. We also computed the global $f(\alpha)$ spectra for the whole $B_{X}$ time series on small and large scales from 10:55 to 11:35 UT, which are depicted by solid red and blue lines, respectively. Deviations from these average $f(\alpha)$ curves classify physical processes occurring during periods A-D. An examination of only the right wings of the distributions leads to the following conlusions (see also Figs. 4a and d):

1. the $f(\alpha)$ spectra estimated on both large and small scales exceed the average $f(\alpha)$ only during period C;

2. during period A (large scale flapping motion), only the large scale (blue circles) exceeds the average blue curve significantly;

3. quiet periods B and D exhibit average or narrower than average distributions.

With the definition of $L I M$, we have introduced a number which quantifies intermittence as an area under the right wing of the $f(\alpha)$ distribution function. We have to emphasize, however, that $f(\alpha)$ distributions cannot be described or replaced by one number. The whole distribution contains more information. It is evident from Fig. 5 that the more intermittent period $\mathrm{C}$ is also characterized by the largest difference between $\alpha_{\max }$ and $\alpha_{\min }$ on small scale (red circles).
Also, only in this case, the maximum of the $f(\alpha)$ curve is significantly shifted to the right. There are multiplicative cascade models for which multifractal distributions of concave shape and the underlying intermittence properties can be described by one parameter, e.g. the P-model (Halsey et al., 1986; Vörös et al., 2002). However, those models cannot fit the data well because of the non-stationarity and shortness of the available time series in the plasma sheet. This is clearly visible in the case of large-scale, non-concave distributions during periods $\mathrm{A}$ and $\mathrm{C}$ (blue circles, Fig. 5). For this reason $L I M$ represents a descriptor which tells more about the intermittent fluctuations than second order statistics, but less than the whole multifractal distribution function.

\subsection{Multi-spacecraft comparison and BBF occurrence}

To facilitate interpretation, the $B_{X}$ components from two Cluster spacecraft (CL1 and CL3) are depicted in Fig. 6a. The difference between the $B_{X}$ components measured at the locations of CL1 and 3 changes substantially during the considered interval, indicating spatial gradients of the order of the distance between CLs within current sheet. The largest spatial gradients occur during and after the flapping motion from 10:55 to 11:10 UT. Large gradients are also present during the interval 11:22-11:30 UT. These two intervals are separated by a $\sim 10 \mathrm{~min}$ interval, from 11:10 to $11: 21 \mathrm{UT}$, characterized by small spatial gradients and $-18<B_{X}<$ $-10 \mathrm{nT}$. Therefore, the spacecraft are outside of the current sheet. There are two more periods when the observed 

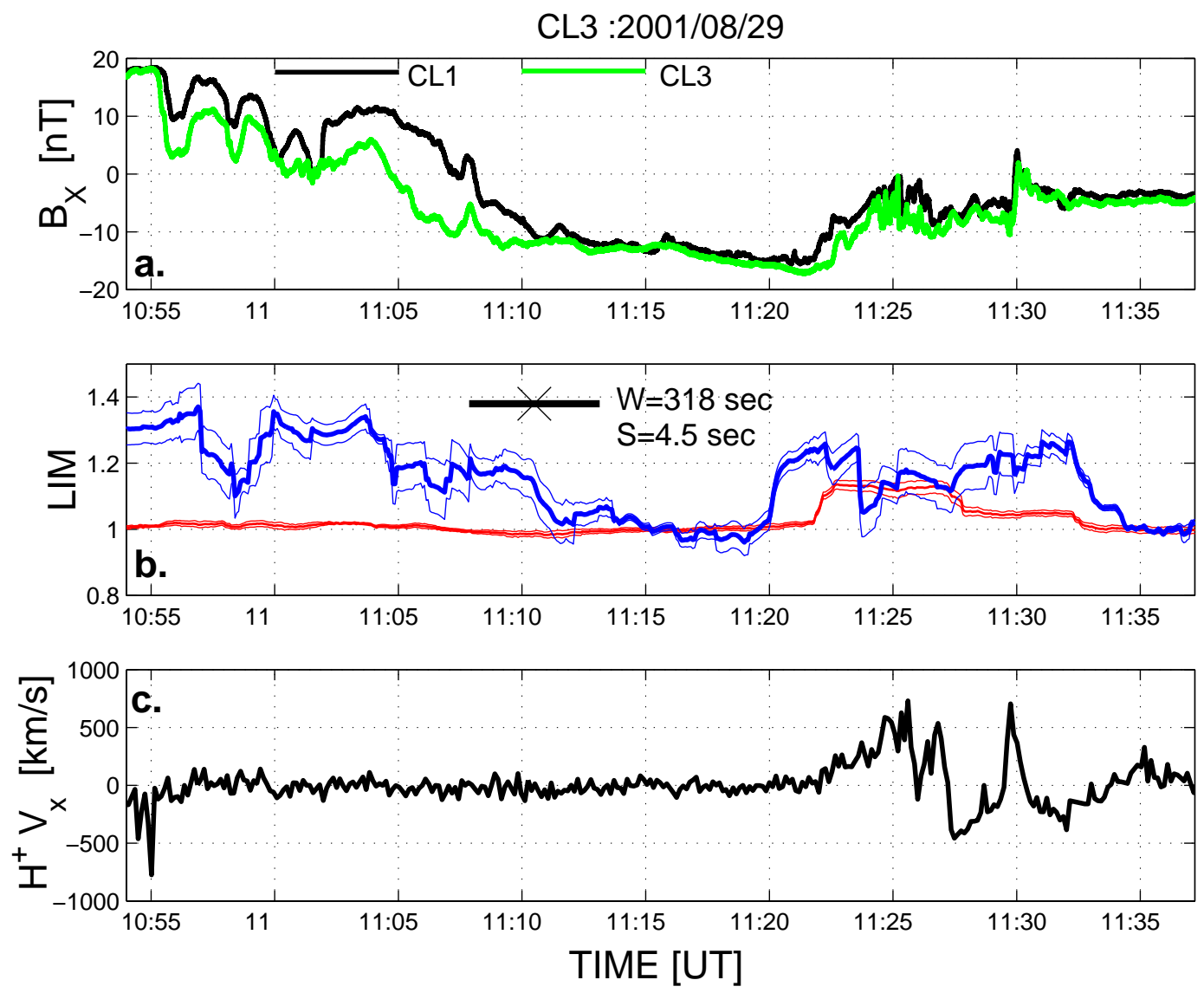

Fig. 6. (a) Magnetic field $B_{X}$ components measured by Cluster 1, 3 spacecraft; (b) $L I M_{L, S}$ for small scales (red line) and large scales (blue line), thin curves show standard deviations; (c) proton velocity data.

spatial gradients are small. The first is before 10:55 UT $\left(B_{X}>18: \mathrm{nT}\right)$, when the spacecraft were in the northern lobe. The interval after 11:30 UT also contains small spatial gradients, but the $B_{X}$ components change from -6 to $2 \mathrm{nT}$, indicating that the spacecraft are closer to the center of current sheet.

Figure 6b shows $L I M_{L, S}$ (red and blue curves). Standard deviations computed from a number of $f(\alpha)$ distributions (Fig. 4d) estimated around $\tau=40$ and $0.4 \mathrm{~s}$ are also depicted by thin lines around $L I M_{L, S}(t)$ in Fig. 6b. Window parameters are also indicated.

It is visible that during the large scale motion (thoroughly analysed by Runov et al., 2002) and after, until $\sim 11: 10$ UT (Fig. 6a), LIM shows enhanced intermittence level on large scales, but not on small scales (Fig. 6b). $L I M_{L}$ is also high before 10:55 UT, only because the local window $W$ extends over the period of wavy motion of the current sheet. As no enhanced intermittence level is observed during the whole interval until $\sim 11: 10$ UT on small scales, we conclude that cross-scale energisation is not present. More precisely, at least in terms of intermittent fluctuations quantified by $L I M$, there was no CSC mechanism present that could couple large-scale energy reservoirs at the level of the MHD flow ( $\sim 40 \mathrm{~s})$ to the small scales $(\sim 0.4 \mathrm{~s})$. We cannot exclude, however, other mechanisms of CSC not directly associated with $L I M$ changes. $L I M_{L}$ tends to decrease rapidly after 11:10 UT because data from outside the current sheet influence its estimation.

Between 11:20 and 11:35 UT, both $L I M_{L}$ and $L I M_{S}$ increase. This enhancement is clearly associated with high frequency intermittent fluctuations in $B_{X}$ (Fig. 6a; see also the global spectrum for period $C$ in Fig. 5) and with the occurence of a BBF. In Fig. $6 \mathrm{c}$ we show the proton velocity data from CIS/CODIF experiment $\left(H^{+} V_{X}\right.$; GSM). Figure 7a shows the magnetic field $B_{Z}$ component of the magnetic field measured by CL3, while Figs. $7 \mathrm{~b}-\mathrm{d}$ show $B_{X}$, proton velocity and $L I M$ at better time resolution than in Fig. 6.

Four windows centered on points marked by crosses indicate the times when $L I M_{L, S}$ significantly increase or decrease relative to the quiet level $\left(L I M_{L, S} \sim 1\right)$. Vertical red and blue arrows indicate the starting points of increase and decrease of $L I M_{L, S}$, respectively.

When the spacecraft enter the current sheet after 11:20 UT, LI $M_{L}$ increases and window 1 shows that the enhancement is associated with the appearance of large-scale fluctuations in $B_{X}$, a small decrease in $B_{Z}$ and gradual increase in $V_{X, H^{+}}$, starting at 11:22:20 UT (see the vertical dashed line at the right end of window 1). Approximately two minutes later, 

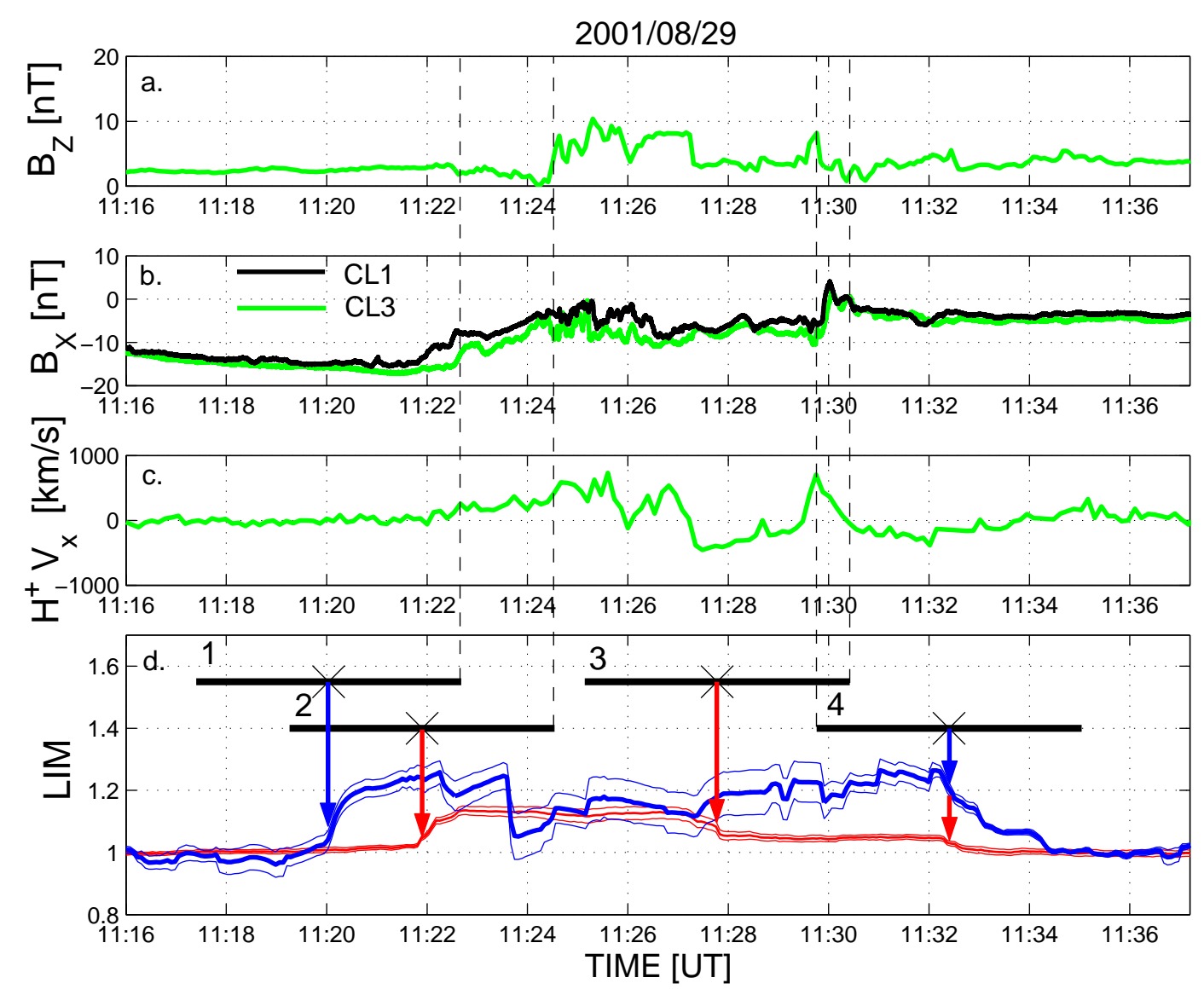

Fig. 7. (a) Magnetic field $B_{Z}$ component from Cluster 3; (b) Magnetic field $B_{X}$ components from Cluster 1, 3; (c) proton velocity data; (d) $L I M_{L, S}$.

the center of window 2 points to the first significant enhancement of $L I M_{S}$ (red vertical arrow). $L I M_{S}$ achieved its maximum value $1.14 \pm 0.02$ within $\sim 40 \mathrm{~s}$. The right end of window 2 is clearly associated with:

1. magnetic field dipolarization (rapid increase in $B_{Z}$ to $\sim 8-10$ nT in Fig. 7a);

2. appearance of high-frequency fluctuations in $B_{X}$ (CL3), (in Fig. 7b);

3. BBF velocities larger than $400 \mathrm{~km} / \mathrm{s}$ (Fig. 7c);

4. enhancements of energetic ion and electron fluxes on CL3 (not shown); all at $\sim 11: 24: 27$ UT.

$L I M_{S}$ drops to $1.05 \pm 0.02$ at 11:27:45 UT (marked by the red arrow from the center of window 3 ). This time, the right end of window 3 starts to leave behind the largest peaks of $V_{X, H^{+}}$, but that is not the only reason for the decrease in $L I M_{S}$. When $L I M_{S}$ decreases, $L I M_{L}$ remains at a high level $(1.24 \pm 0.05)$, or even increases, due to the sudden jump in $B_{X}$ from -10 to $+2 \mathrm{nT}$ closely before 11:30 UT. It was previously mentioned that after 11:30 UT, the spacecraft moved closer to the center of the current sheet. Therefore, we suppose that, due to the large-scale motion of the current sheet, which keeps $L I M_{L}$ at a high level, the spacecraft appear to be outside of the region of BBF-associated turbulence. This is also supported by the simultaneous decrease in both $L I M_{L}$ and $L I M_{S}$ at approximately 11:32:30 UT, when window 4 includes $B_{X}$ from the region with small gradients after 11:30 UT. Therefore, during the interval between the right ends of window 2 and 3, i.e. within a time period of $\sim 6 \mathrm{~min}$ from $\sim 11: 24$ to $\sim 11: 30 \mathrm{UT}, L I M$ analysis indicates BBF and dipolarization-associated CSC between MHD and small, possibly kinetic scales. An alternative to the CSC might be a simultaneous, but independent enhancement of intermittent fluctuations on both large and small scales. As was mentioned earlier, an identification of the energy-cascading process is almost impossible using the applied method. The primary pile-up of energy associated with the increase in BBF velocity on large scales at 11:22:20 UT, however, seems to indicate that in this case, small-scale fluctuations are energised by MHD scale rapid flows. Unambiguous evidence for or against BBF-related CSC requires a statistical ensemble of events to be analysed. We mention that inverse cascades during current disruption events were reported by Lui (1998).

The large difference between $L I M_{L}$ and $L I M_{S}$ after 11:28 UT can be attributed to the prevailing large-scale motion of the current sheet. The spacecraft moved closer to the center of the current sheet, where the multiscale $L I M$ signs 
of CSC are already absent. This can be explained by the transitory and localized nature of CSC.

\section{Conclusions}

We proposed a windowed multifractal method to quantify local intermittence of magnetic field fluctuations obtained by Cluster. The main results of this paper comprise a multi-scale description of large-scale current sheet motion and of a BBFassociated cross-scale energisation process. We have shown as Cluster passes through different plasma regions, physical processes exhibit non-stationary intermittence properties on MHD and small, possibly kinetic scales. As any robust estimation of turbulence characteristics requires processing of long time series (due to the presence of energetic but rare events), the observed transitory and non-stationary nature of fluctuations prevents us from unambiguously supporting or rejecting a model for plasma sheet turbulence.

The multifractal description of intermittent magnetic fluctuations is in accordance with previous knowledge that the change in fractal scaling properties can be associated with phase transition-like phenomenon and self organization in the plasma sheet (Chang, 1999; Consolini and Chang, 2001; Consolini and Lui, 2001; Milovanov et al., 2001). Our results also support the idea of Angelopoulos et al. (1999a) that BBF-related intermittent turbulence may represent an effective way for CSC. Propagating BBFs can modify a critical threshold for nonlinear instabilities or trigger further localized reconnections due to the free energy present on multiple scales. In this sense, our results suggest that BBFs may represent those multiscale carriers of energy, flux and momentum, which lead to the avalanche-like spread of disturbances on medium or large scales (Klimas et al., 2000; Lui, 2002). In this respect classification of multiscale physical processes using $L I M$, or multifractal distributions offers a way in which the role of turbulence in a variety of dynamical processes within the plasma sheet can be statistically evaluated.

Acknowledgements. The authors acknowledge the use of FRACLAB package developed at the Institut National de Recherche en Informatique, France. ZV thanks A. Petrukovich for many valuable suggestions. The work by KHG was financially supported by the German Bundesministerium für Bildung und Wissenschaft and the German Zentrum für Luft- und Raumnfahrt under contract $50 \mathrm{OC}$ 0103.

Topical Editor T. Pulkkinen thanks A. Klimas and another referee for their help in evaluating this paper.

\section{References}

Angelopoulos, V., Baumjohann, W., Kennel, C. F., Coroniti, F. V., Kivelson, M. G., Pellat, R., Walker, R. J., Lühr, H., and Paschmann, G.: Bursty bulk flows in the inner central plasma sheet, J. Geophys. Res., 97, 4027-4039, 1992.

Angelopoulos, V., Mukai, T., and Kokubun, S.: Evidence for intermittency in Earth's plasma sheet and implications for selforganized criticality, Phys. Plasmas, 6, 4161-4168, 1999a.
Angelopoulos, V., Mozer, F. S., Lin, R. P., Mukai, T., Tsuruda, K., Lepping, R., and Baumjohann, W.: Comment on "Geotail survey of ion flow in the plasma sheet: Observations between 10 and $50 R_{E}$ " by W. R. Paterson et al., J. Geophys. Res., 104, $17521-$ $17525,1999 \mathrm{~b}$.

Balogh, A., Carr, C. M., Acuña, M. H., et al.: The Cluster magnetic field investigation: overview of in-flight performance and initial results, Ann. Geophysicae, 19, 1207-1217, 2001.

Baumjohann, W., Paschmann, G., and Lühr, H.: Characteristics of high-speed ion flows in the plasma sheet, J. Geophys. Res., 95, 3801-3810, 1990.

Borovsky, J. E., Elphic, R. C., Funsten, H. O., and Thomsen, M. F.: The Earth's plasma sheet as a laboratory for flow turbulence in high- $\beta$ MHD, J. Plasma Phys., 57, 1-34, 1997.

Canus, Ch., Véhel, J. L., and Tricot, C.: Continuous large deviation multifractal spectrum: definition and estimation, Proc. Fractals 98, Malta, 1998.

Consolini, G. and Chang, T. S.: Magnetic field topology and criticality in geotail dynamics: relevance to substorm phenomena, Space Sci. Rev., 95, 309-321, 2001.

Consolini, G. and Lui, A. T. Y.: Symmetry breaking and nonlinear wave-wave interaction in current disruption: possible evidence for a phase transition, AGU Monograph on Magnetospheric Current Systems, 118, 395-401, 2001;

Chang, T.: Self-organized criticality, mult-fractal spectra, sporadic localized reconnections and intermittent turbulence in the magnetotail, Phys. Plasmas, 6, 4137-4145, 1999.

Chang, T., Wu, Ch., and Angelopoulos, V.: Preferential acceleration of coherent magnetic structres and bursty bulk flows in Earth's magnetotail, Phys. Scripta, T98, 48-51, 2002.

Frisch, U.: Turbulence, Cambridge University Press, 1995.

Horbury, T. S.: Cluster II analysis of turbulence using correlation functions, in Proc. Cluster II Workshop, ESA SP-449, 89-97, 2000.

Halsey, T. C., Jensen, M. H., Kadanoff, L. P., Procaccia, I., and Shraiman, B. I.: Fractal measures and their singularities: the characterization of strange sets, Phys. Rev. A, 33, 1141-1151, 1986.

Klimas, A. J., Valdivia, J. A., Vassiliadis, D., Baker, D. N., Hesse, M., and Takalo, J.: Self-organized criticality in the substorm phenomenon and its relation to localized reconnection in the magnetospheric plasma sheet, J. Geophys. Res., 105, 18765-18 780, 2000.

Lui, A. T. Y.: Multiscale and intermittent nature of current disruption in the magnetotail, Phys. Space Plasmas, 15, 233-238, 1998.

Lui, A. T. Y.: Multiscale phenomena in the near-Earth magnetosphere, J. Atmosph. Sol. Terr. Phys., 64, 125-143, 2002.

Milovanov, A. V., Zelenyi, L. M., Zimbardo, G., and Veltri, P.: Selforganized branching of magnetotail current systems near the percolation threshold, J. Geophys. Res., 106, 6291-6307, 2001.

Nakamura, R., Baumjohann, W., Schödel, R., Brittnacher, M., Sergeev, V. A., Kubyshkina, M., Mukai, T., and Liou, K.: Earthward flow bursts, auroral streamers, and small expansions, J. Geophys. Res., 106, 10 791-10 802, 2001.

Neagu, E., Borovsky, J. E., Thomsen, M. F., Gary, S. P., Baumjohann, W., and Treumann, R. A.: Statistical survey of magnetic field and ion velocity fluctuations in the near-Earth plasma sheet: Active Magnetospheric Particle Trace Explorers / Ion Release Module (AMPTE/IRM) measurements, J. Geophys. Res., 107, 10.1029/2001JA000318, 2002.

Rème, H., Aoustin, C., Bosqued, J. M., et al.: First multispacecraft ion measurements in and near the Earth's magnetosphere with 
the identical Cluster ion spectrometry (CIS) experiment, Ann. Geophysicae, 19, 1303-1354, 2001.

Riedi, R. H.: Multifractal processes, Technical Report, TR99-06, Rice University 1999.

Runov, A., Nakamura, R., Baumjohann, W., Zhang, T. L., Volwerk, M., Eichelberger, H. U., and Balogh, A.: Cluster observation of a bifurcated current sheet, Geophys. Res. Lett., in press, 2002.

Schödel, R., Baumjohann, W., Nakamura, R., Sergeev, V. A., and Mukai, T.: Rapid flux transport in the central plasma sheet, J. Geophys. Res., 106, 301-313, 2001.
Tetreault, D.: Turbulent relaxation of magnetic fields 2. Selforganization and intermittency, J. Geophys. Res., 97, 8541-8547, 1992.

Tsinober, A.: An informal introduction to turbulence, Kluwer Academic Publishers, 2001.

Véhel, J. L. and Vojak, R.: Multifractal analysis of Choquet capacities: preliminary results, Adv. Appl. Math., 20(1), 1-43, 1998.

Vörös, Z., Jankovičová, D., and Kovács, P.: Scaling and singularity characteristics of solar wind and magnetospheric fluctuations, Nonlin. Proc. Geophys., 9, 149-162, 2002. 